\title{
Complications of Decompressive Craniectomy
}

\author{
Andrew Margules, BA ${ }^{1}$, Jack Jallo, MD, $\mathrm{PhD}^{2}$ \\ ${ }^{1}$ Jefferson Medical College, Thomas Jefferson University, Philadelphia, PA \\ 2Department of Neurological Surgery, Thomas Jefferson University, Philadelphia, PA
}

\section{Introduction}

Management of intracranial hypertension is often the greatest challenge for a neurosurgeon treating a patient who has suffered a severe traumatic brain injury (TBI). Too sharp a rise in intracranial pressure (ICP) can overwhelm the brain's ability to regulate cerebral blood flow (CBF). The resulting cerebral ischemia can contribute to diminished function or to death. Conventional treatment options, such as hyperosmolar dehydration, hyperventilation and barbiturate coma, form the first line of defense. However, it has been reported that $10-15 \%$ of patients with intracranial hypertension resulting from severe TBI do not respond to maximal medical management. ${ }^{1}$ In these refractory cases, decompressive craniectomy may provide a lifesaving reduction in ICP.

Because intracranial volume is fixed, any increase in its contents (through swelling, edema, hemorrhage, mass lesions, or other means) must produce an increase in pressure. Decompressive craniectomy allows for intracranial volume expansion, thereby avoiding intracranial hypertension. A recent study reported that decompressive craniectomy was effective in lowering ICP to safe levels (below $20 \mathrm{mmHg}$ ) in $80 \%$ of cases. ${ }^{2}$ In addition, several studies have found that the reduction in ICP following this procedure can contribute to improvements in cerebral compliance, cerebral oxygen supply, cerebral blood perfusion, and various CT signs. ${ }^{3}$ One hospital found that attempts to remove the hematoma often caused far more damage than simply controlling ICP and allowing the hematoma to resolve on its own, and made decompressive craniectomies routine following contusion hemorrhage as part of a policy to "not mess with a swollen brain."4

Despite increasing popularity, however, decompressive craniectomy remains a controversial treatment option, with strong advocates and opponents. There is virtually no definitive evidence from controlled studies to establish that the procedure actually improves long-term outcome following severe TBI. While many studies have found significant benefits to certain patients, mortality rates following decompressive craniectomy for severe TBI are high. In one 5-year study involving 170 decompressive craniectomies, $50 \%$ of patients died. ${ }^{5}$

Interestingly, the procedure itself is considered to present relatively little risk, with low reported occurrence of surgical complications. ${ }^{6}$ Rather, morbidity and mortality are chiefly associated with late complications secondary to surgical decompression. Many of these complications arise from the normal pathophysiological alterations in cerebral compliance, CBF, autoregulation and CSF circulation that occur following removal of a large piece of the skull. ${ }^{5}$ Among the more well recognized complications associated with decompressive craniectomy are expansion of hemorrhagic contusions, external cerebral herniation, subdural hygroma, infection, hydrocephalus, syndrome of the trephined and epilepsy. These and other complications, if they arise, tend to do so along fairly consistent time points following decompression. Yang et al. reported that 54 of 108 patients (50\%) developed complications related to surgical decompression, including 28 patients (25.9\%) who developed more than one complication. ${ }^{4}$ Adding to this risk is the eventual need for a second operation, a cranioplasty, to replace the bone flap. Gooch et al., reported that 21 of 62 patients (34\%) undergoing this reconstruction experienced complications. ${ }^{7}$

Not unexpectedly, there seems to be an association between the severity of the initial injury, measured by the Glasgow Coma Scale (GCS), and the outcome of decompressive craniectomy. Yang et al. found patients with worse GCS score had higher complication rates and worse outcomes. ${ }^{3}$ Other studies have found similar correlations between GCS score and prognosis, measured by the Glasgow Outcome Score (GOS). ${ }^{3,8,9}$

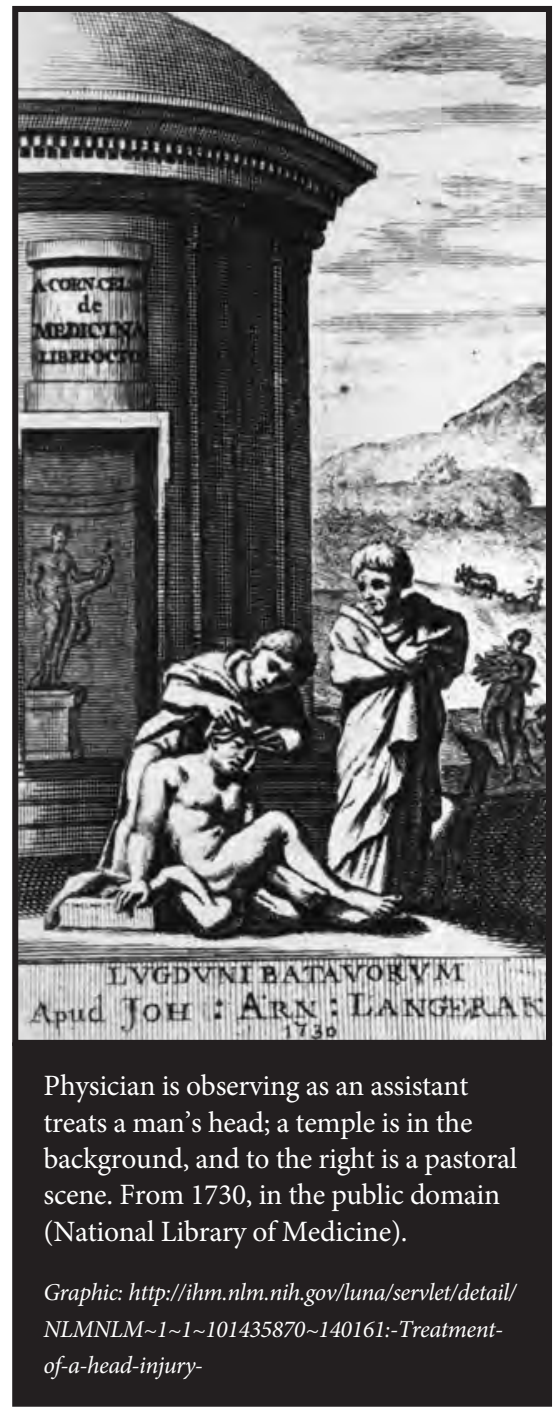

This work is a review of the risk factors and underlying mechanisms of the complications associated with decompressive craniectomy. The focus is on balancing the potential life-saving benefits of craniectomy with the concerns about the quality of life of survivors.

\section{Complications}

Expansion of Hemorrhagic Contusions Delayed development or expansion of hemorrhagic contusions is a frequent and typically inauspicious complication of TBI. It is the most common cause of deterioration 
and death in patients who experienced a lucid period following severe TBI. ${ }^{10}$ One study of patients receiving One study at a Level one Trauma Center, of patients receiving two head CTs without intervening surgery, found that 86 of 229 acute hemorrhages (38\%) grew between the first and second scans. ${ }^{11}$ In contrast, Flint et al. found a substantially higher incidence following decompressive craniectomy, as new or expanded contusions occurred in 23 of 40 patients (58\%). $81.5 \%$ of this new hemorrhage volume was ipsilateral to the hemicraniectomy. ${ }^{2}$

One theory explaining this phenomenon is that bone removal in decompressive craniectomy could lead to loss of tamponade effect, facilitating hematoma expansion both ipsilaterally and on rare occasions contralaterally. ${ }^{5}$

Flint et al. found a strong correlation between the Rotterdam score, a measure of TBI severity based on initial head CT, and the later development of new or expanded hemorrhages after craniectomy. The authors concluded that higher Rotterdam Scores are predictive of increased risk of contusion expansion, and that the volume of expansion following hemicraniectomy is in turn associated with mortality and poor outcome.

Most intracranial hematoma expansions occur perioperatively or early after the first operation. The key to management is early detection and intervention, and those that occur postoperatively will likely require reoperation. ${ }^{3}$ Yang et al. advise routine CT scanning immediately after decompressive craniectomy, another CT within $24 \mathrm{~h}$, and serial scans over the next week.

\section{External Cerebral Herniation}

Herniation of swollen brain tissue through the craniectomy defect is often observed following decompression. This may lead to pinching of cortical veins or laceration of brain tissue at the defect edge, resulting in ischemia and necrosis of herniated tissue. As a result, patients with signs of herniation have been reported to have significantly worse outcomes than those without these signs. ${ }^{12}$ This increased swelling following decompressive craniectomy may be a consequence of hyperperfusion of brain tissue, or of increased transcapillary leakage of edema fluid resulting from the drop in interstitial hydrostatic pressure. ${ }^{5}$

Yang et al. reported herniation in 30 of 108 decompressive craniectomy patients (27.8\%). Notably, this complication was more pronounced in patients for whom the procedure was performed with small-sized craniectomy. ${ }^{3}$ This led the authors to conclude that this complication may be minimized by performing a sufficiently large craniectomy, allowing the brain to expand outward without constriction. Other potential management tools include augmentative duraplasty to limit cephalocele, or insertion of "vascular cushions" adjacent to large draining veins at craniectomy margin to reduce risk of venous ischemia. $^{5}$

\section{Subdural Hygromas}

Subdural hygroma formation is the most commonly described complication following decompressive craniectomy, ${ }^{13}$ however the mechanism through which the CSF comes to accumulate in the subdural space is a topic of disagreement. The most commonly implicated cause is altered CSF circulation dynamics following decompression, however other authors have suggested increased cerebral perfusion pressure ${ }^{14}$ or injury to the arachnoid-dura interphase layer as a result of trauma or iatrogenic surgical disruption ${ }^{15}$ as the link between hygromas and decompressive craniectomy.

Incidence of subdural hygroma following decompressive craniectomy, while not firmly established, are consistently high. Yang et al., documented 23 cases of hygromas in 108 patients (21.3\%), ${ }^{3}$ and Aarabi et al., reported the complication in 25 of 50 patients (50\%) in their 2006 study.

The most comprehensive analysis of subdural hygromas secondary to decompressive craniectomy was done by Aarabi et al. in 2009. They saw hygromas develop in 39 of 68 patients (57.4\%) who had survived beyond One month after TBI and the decompression procedure. Whereas the accumulation of CSF in the subdural space normally peaks at 20-30 days in closed head injuries, they found that following decompressive craniectomies, hygromas could be seen as early as the second postoperative day. They observed that the fluid collections could increase in size for up to four weeks, and could linger for as long as four months after surgery.

While most hygromas ultimately resolve spontaneously, there are negative symptoms and greater risks associated with them that may demand more proactive treatment. One major concern is the potential for mass effect, when the hygroma causes a shift in midline structures that can precipitate cognitive decline. Aarabi et al. found that while hygromas appearing contralaterally to the side of decompression were quite rare $(8 \%$ of hygromas found in study), there seemed to be a tendency for these to cause more symptoms. ${ }^{15}$ Additionally, there are concerns over the potential evolution of a benign hygroma into a more severe and chronic subdural hematoma, which will require surgical evacuation. A possible explanation for this is that increased protein content in the hygroma fluid triggers an inflammatory response. This leads to the formation of a neomembrane surrounding the hygroma with fragile and leaky capillaries. It has been reported that this transformation occurs in 16.7-32.8\% of all traumatic subdural hygromas. ${ }^{16}$

Several steps can be taken in the prevention and management of subdural hygromas. Augmentative duraplasty at the time of decompression has been observed to lower the incidence of hygroma formation. ${ }^{5}$ The majority of hygromas gradually disappear without surgical intervention. Contralateral hygromas, however, because of their greater tendency to cause a midline shift, may require more aggressive treatment, such as bur hole evacuation, bedside subdural drains, or craniotomy. ${ }^{15}$ Feng et el. suggest that antiinflammatory agents could potentially be helpful in stemming the evolution of hygromas into subdural hematomas. ${ }^{16}$

\section{Infection}

With the administration of intraoperative antibiotics, postoperative infection rates following decompressive craniectomy should not be more than the 3-7\% typically reported in neurosurgical literature. ${ }^{1,15}$ Most studies reviewed were consistent with this. ${ }^{3,4,15,17}$

The infection rates are low in spite of several factors associated with decompressive craniectomy that may lead one to expect higher incidence. The typical incision is a large reverse question mark with a long scalp pedicle on a comparatively small base, predisposing it to wound breakdown along the parietal and posterior temporal limbs farthest along the flap. ${ }^{5}$ Additionally, expansive duraplasty with a dural substitute has been associated with an increased chance of infection. ${ }^{1}$ With these concerns in mind, meticulous and watertight expansive duraplasty and scalp closure may be necessary to prevent egress of CSF from the skin edges and limit the risk of deep central nervous system infection. ${ }^{15}$

The greatest risk of infection is associated with the cranioplasty to replace the bone flap. Repeat surgery inherently increases the risk of infection, but cranioplasty in particular 
has been associated with a higher infection rate than craniectomy. ${ }^{7}$ The use of autologous bone graft materials has shown decreased risk of infection as compared to various substitute materials. $^{18}$ At the same time, however, storage of bone in a freezer for prolonged periods of time before re-implantation appears to increase the risk of infection. ${ }^{5}$ Neurosurgeons are thus faced with something of a conundrum, as convention states that shorter time from craniectomy to cranioplasty is associated with poorer outcome. Many are starting to suggest, however, that the rationale for this waiting period, based on a 1979 study involving patients with penetrating head injuries, may not translate to patients who have undergone decompressive craniectomy in the setting of a non-penetrating injury. ${ }^{\text {? }}$ This theory was supported in a recent study by Chang et al., which found that patients undergoing cranioplasty within three months of craniectomy had a significantly lower overall complication rate than those waiting longer than six months, and a lower rate tending towards significance in those waiting four to six months. ${ }^{18}$

\section{Post-traumatic Hydrocephalus}

The reported incidence of post-traumatic hydrocephalus (PTH) in patients with head trauma range from $0.7-86 \%{ }^{19}$ This wide variation is largely due to inconsistent diagnostic criteria and classification of hydrocephalus. ${ }^{3}$ PTH is an active and progressive process characterized by the accumulation of excessive cerebrospinal fluid due to liquorodynamic disturbances following craniocerebral injury. ${ }^{19}$ It is therefore unsurprising that decompressive craniectomy is a further risk factor for the development of PTH, given the alterations in CSF flow dynamics that often follow decompression.

Determining the association between decompressive craniectomy and PTH difficulties confounded by the fact that several of the aforementioned complications of decompression, such as hemorrhage and infection, are themselves independent risk factors for the development of hydrocephalus. In a study which attempted to control for these factors, Choi et al. reported an incidence of PTH of $4.0 \%$ among patients receiving conservative, non-decompressive treatment, and 20.7\% among patients who underwent decompression. Other studies reported rates of PTH following decompression ranging from 7.0-
$12.9 \%{ }^{3,4,15}$ These data, however are difficult to interpret due the lack of non-decompressive controls.

Proposed mechanisms regarding the postcraniectomy CSF flow abnormalities linked to hydrocephalus most often involve the arachnoid granulations, pressure-dependent one-way valves through which CSF drains from the subarachnoid space into venous sinuses. These channels could potentially be obstructed by inflammation or mechanical blockage by post surgical debris. ${ }^{19}$ Waziri et al. reported a "flattening" of the normally dicrotic ICP waveform following decompressive craniectomy, presumably resulting from the transmission of pulse pressure out of the open cranium ${ }^{20}$.

If this were true, then early cranioplasty should lead to the restoration of normal ICP dynamics and spontaneous alteration of hydrocephalus. Waziri et al found this to indeed be the case for a portion of patients. ${ }^{20}$ The appropriate timing of cranioplasty, however, is largely dictated by the degree of residual swelling. Bone flap replacement without an adequate decrease in swelling is associated with a higher risk of developing brain edema and infection. ${ }^{19}$ Therefore, in the case of more severe brain injury, delayed cranioplasty may be necessary, predisposing these patients to the development of hydrocephalus. ${ }^{20}$

Treatment of PTH through CSF shunting is difficult to predict, and generally the results are not encouraging. Additionally, shunt treatment for hydrocephalus in the setting of a large cranial defect may increase the risk of neurological deterioration consistent with paradoxical herniation. ${ }^{5}$ Fortunately, in clinical practice, even when incidence of PTH are relatively high, only $1-4 \%$ of these patients eventually require shunt operation. ${ }^{19}$

\section{Syndrome of the Trephined}

Syndrome of the Trephined, also known as sinking skin flap syndrome, is a delayed complication of decompressive craniectomy, with symptoms often appearing weeks to months after the procedure. ${ }^{5}$ Symptoms commonly include headaches, dizziness, irritability, concentration difficulty, memory problems and mood disturbances. Some studies have also described delayed motor deficits associated with the condition, referred to as "motor trephine syndrome." ${ }^{21}$ The diagnosis of this condition is often overlooked, as many of the symptoms are also commonly associated with post-concussion and post-traumatic stress syndromes that tend to accompany traumatic brain injury. ${ }^{5}$

The mechanisms underlying the development of these symptoms are still the subject of debate. One theory describes the sinking of the scalp overlying the cranial defect, which, without bone support, transmits atmospheric pressure directly onto the surface of the brain. This reduces the subarachnoid space and presses against the underlying cortex, resulting in turbulent CSF circulation and decreased cortical blood perfusion. ${ }^{3}$ Another theory attributes these symptoms to two factors. First, parenchymal injury elicits fluid shifts into the brain tissue and decreased resistance at the craniectomy site allows fluid accumulation in the area underlying the defect. Second, decompensated CSF flow can, at late points, manifest as leakage of CSF and edema fluid into vulnerable areas of the injured parenchyma underlying the defect. Both of these factors could lead to impaired cortical blood flow to the region underlying the defect. ${ }^{5}$

Following decompressive craniectomy, patients should be monitored for the neurological, psychiatric and motor symptoms of Trephined. These deficits are usually reversible, and often resolve following cranioplasty. ${ }^{3}$ For this reason, early cranioplasty is a treatment option, but only in the context of the potential risks associated with premature return of the bone flap.

\section{Delayed Paradoxical Herniation}

There are well established hazards associated with lumbar puncture in patients with elevated ICP, due to the risk of herniation of the brain through the foramen magnum. Interestingly, there appears to be a similar, though sparsely reported, risk associated with lumbar puncture in patients having recently undergone surgical decompression. The suggested mechanism involves the establishment of a negative pressure in the spinal canal due to spinal CSF drainage, coupled with the effects of gravity and atmospheric pressure on the exposed brain. This allows the brain to shift towards the infratentorial compartment, causing herniation and brainstem compression and resulting in neurologic deterioration ${ }^{22}$.

Based on this, there is commonly a very high threshold for the performance of a lumbar puncture in patients with a decompressive craniectomy. Recommended therapy for delayed paradoxical herniation has been to position the patient's head down (Trendelenburg positioning), administer intravenous fluids, and clamp CSF drainage. ${ }^{5}$ It has been suggested 
that these measures alone may be inadequate to resolve some cases of paradoxical herniation, and administration of a lumbar blood patch should be part of the recommended treatment. ${ }^{22}$ Early cranioplasty may also correct the problem, but not everyone is a candidate and the requisite risks must be taken into account. ${ }^{5}$

\section{Bone Resorption}

Resorption of autologous bone flaps following cranioplasty is common, with reported incidence as high as $50 \% .{ }^{15}$ Techniques suggested to avoid bone resorption include tight approximation of the free flap to a lengthy surface of the native craniectomy margin, rigid plate and and screw fixation to limit flap movement, and upfront synthetic cranioplasty repair when multiple fragments are involved. ${ }^{5}$ It has been suggested that osteoclast inhibitors may also be a method of preventing bone resorption..$^{23}$

\section{Loss of Function}

Reports of high rates of unfavorable outcome raise the concern that decompressive craniectomy may rescue patients lives only to leave them with severe disability or perhaps even in a persistent vegetative state. ${ }^{25}$ Other studies have challenged this conclusion, suggesting that the average survivor of decompressive craniectomy is left with only moderate disability. ${ }^{8}$ These discrepancies highlight the fact that no matter how well ICP is controlled, we still lack an adequate understanding of the longterm efficacy of decompressive craniectomy as a tool for improving functional outcome following traumatic brain injury. Further study into the specific physiological effects of decompression and the associations of various prognostic factors with functional outcome is necessary to better guide patient selection.

\section{Conclusions}

Some of the complications of decompressive craniectomy may be unavoidable, as they are associated with the precipitating traumatic brain injury or with the normal physiologi- cal changes that occur following removal of a large piece of the skull. These risks can be minimized, however, as we improve strategies of patient selection and complication management, and as we gain a better understanding of the pathophysiological processes underlying the complications. Controlling these risks will establish the feasibility of decompressive craniectomy as a treatment for medically refractive increases in intracranial pressure.

1. Aarabi B, Hesdorffer DC, Ahn ES, Aresco C, Scalea TM, Eisenberg HM. Outcome following decompressive craniectomy for malignant swelling due to severe head injury. J Neurosurg. 2006 Apr;104(4):469-79

2. Flint AC, Manley GT, Gean AD, Hemphill JC 3rd, Rosentha G. Post-operative expansion of hemorrhagic contusions after unilateral decompressive hemicraniectomy in severe traumatic brain injury. J Neurotrauma. 2008 May;25(5):503-12

3. Yang XF, Wen L, Shen F, Li G, Lou R, Liu WG, Zhan RY. Surgical complications secondary to decompressive craniectomy in patients with a head injury: a series of 108 consecutive cases. Acta Neurochir (Wien). 2008 Dec;150(12):1241-7; discussion 1248. Epub 2008 Nov 13.

4. Huang AP, Tu YK, Tsai YH, Chen YS, Hong WC, Yang CC, Kuo LT, Su IC, Huang SH, Huang SJ. Decompressive craniectomy as the primary surgical intervention for hemorrhagic contusion. J Neurotrauma. 2008 Nov;25(11):1347-54.

5. Stiver SI. Complications of decompressive craniectomy for traumatic brain injury. Neurosurg Focus. 2009 Jun;26(6):E7.

6. Kilincer C, Simsek O, Hamamcioglu MK, Hicdonmez T, Cobanoglu S. Contralateral subdural effusion after aneurysm surgery and decompressive craniectomy: case report and review of the literature. Clin Neurol Neurosurg. 2005 Aug;107(5):412-6

7. Gooch MR, Gin GE, Kenning TJ, German JW. Complications of cranioplasty following decompressive craniectomy: analysis of 62 cases. Neurosurg Focus. 2009 Jun;26(6):E9.

8. Danish SF, Barone D, Lega BC, Stein SC. Quality of life after hemicraniectomy for traumatic brain injury in adults. A review of the literature. Neurosurg Focus. 2009 Jun;26(6):E2.

9. Ucar T, Akyuz M, Kazan S, Tuncer R. Role of decompressive surgery in the management of severe head injuries: prognostic factors and patient selection. J Neurotrauma. 2005 Nov;22(11):1311-8

10. Gudeman SK, Kishore PR, Miller JD, Girevendulis AK, Lipper MH, Becker DP. The genesis and significance of delayed traumatic intracerebral hematoma. Neurosurgery. 1979 Sep;5(3):309-13

11. Chang EF, Meeker M, Holland MC. Acute traumatic intraparenchymal hemorrhage: risk factors for progression in the early post-injury period. Neurosurgery. 2007 Jul;61(1 Suppl):222-30; discussion 230-1.

12. Woertgen C, Rothoerl RD, Schebesch KM, Albert R. Comparison of craniotomy and craniectomy in patient with acute subdural haematoma. J Clin Neurosci. 2006 Aug;13(7):718-21. Epub 2006 Aug 14
13. Kakar V, Nagaria J, John Kirkpatrick P. The current status of decompressive craniectomy. Br J Neurosurg. 2009 Apr;23(2):147-57

14. Lang JK, Ludwig HC, Mursch K, Zimmerer B, Markakis E. Elevated cerebral perfusion pressure and low colloid osmotic pressure as a risk factor for subdural space-occupying hygromas?. Surg Neurol. 1999 Dec;52(6):630-7.

15. Aarabi B, Chesler D, Maulucci C, Blacklock T, Alexander M. Dynamics of subdural hygroma following decompressive craniectomy: a comparative study. Neurosurg Focus. 2009 Jun;26(6):E8.

16. Feng JF, Jiang JY, Bao YH, Liang YM, Pan YH. Traumatic subdural effusion evolves into chronic subdural hematoma: two stages of the same inflammatory reaction?. Med Hypotheses. 2008;70(6):1147-9. Epub 2008 Jan 14.

17. Jiang JY, Xu W, Li WP, Xu WH, Zhang J, Bao YH, Ying YH, Luo QZ. Efficacy of standard trauma craniectomy for refractory intracranial hypertension with severe traumatic brain injury: a multicenter, prospective, randomized controlled study. J Neurotrauma. 2005 Jun;22(6):623-8

18. Chang V, Hartzfeld P, Langlois M, Mahmood A, Seyfried D. Outcomes of cranial repair after craniectomy. J Neurosurg. 2009 Jul 17. [Epub ahead of print]

19. Choi I, Park HK, Chang JC, Cho SJ, Choi SK, Byun BJ. Clinical factors for the development of posttraumatic hydrocephalus after decompressive craniectomy. J Korean Neurosurg Soc. 2008 May;43(5):227-31. Epub 2008 May 20

20. Waziri A, Fusco D, Mayer SA, McKhann GM 2nd, Connolly ES Jr. Postoperative hydrocephalus in patients undergoing decompressive hemicraniectomy for ischemic or hemorrhagic stroke. Neurosurgery. 2007 Sep;61(3):489-93; discussion 493-4.

21. Stiver SI, Wintermark M, Manley GT. Reversible monoparesis following decompressive hemicraniectomy for traumatic brain injury. J Neurosurg. 2008 Aug;109(2):245-54.

22. Vilela MD. Delayed paradoxical herniation after a decompressive craniectomy: case report. Surg Neurol. 2008 Mar;69(3):293-6; discussion 296. Epub 2007 Jun 21

23. Väänänen K. Mechanism of osteoclast mediated bone resorption--rationale for the design of new therapeutics. Adv Drug Deliv Rev. 2005 May 25;57(7):959-71. Epub 2005 Apr 15

24. Timofeev I, Hutchinson PJ. Outcome after surgical decom pression of severe traumatic brain injury. Injury. 2006 Dec;37(12):1125-32. Epub 2006 Nov 1.

25. Uçar T, Akyuz M. Management of severe traumatic brain injury by decompressive craniectomy. Neurosurgery. 2001 Oct;49(4):1022.

26. Ho CL, Wang CM, Lee KK, Ng I, Ang BT. Cerebral oxygenation, vascular reactivity, and neurochemistry following decompressive craniectomy for severe traumatic brain injury. J Neurosurg. 2008 May;108(5):943-9.

27. Howard JL, Cipolle MD, Anderson M, Sabella V, Shollenberger D, Li PM, Pasquale MD. Outcome after decompressive craniectomy for the treatment of severe traumatic brain injury. J Trauma. 2008 Aug;65(2):380-5; discussion 385-6

28. Morgalla MH, Will BE, Roser F, Tatagiba M. Do long-term results justify decompressive craniectomy after severe traumatic brain injury?. J Neurosurg. 2008 Oct;109(4):685-90. 